Review

\title{
The role of Exosomes in the Pathogenesis of Nasopharyngeal Carcinoma and the involved Clinical Application
}

\author{
Huidan Luo, Bin $\mathrm{Yi}^{凶}$ \\ Department of Clinical Laboratory, Xiangya Hospital, Central South University, Changsha, Hunan Province 410008, China. \\ $\triangle$ Corresponding author: Bin Yi, PhD, Department of Clinical Laboratory, Xiangya Hospital, Central South University, Changsha, Hunan Province 410008, \\ China. Tel: +86-13707495781; Fax: +86-731-84327332; E-mail: xyyibin@163.com. \\ (c) The author(s). This is an open access article distributed under the terms of the Creative Commons Attribution License (https://creativecommons.org/licenses/by/4.0/). \\ See http://ivyspring.com/terms for full terms and conditions.
}

Received: 2021.02.22; Accepted: 2021.05.17; Published: 2021.05.27

\begin{abstract}
Exosomes are nanoscale membrane vesicles, which carry biologically active substances of their cell of origin and play an important role in signal transduction and intercellular communication. At present, exosomes have been identified as a promising non-invasive liquid biopsy biomarker in the tissues and circulating blood of nasopharyngeal carcinoma (NPC) and found to participate in regulating pathophysiological process of the tumor. We here review recent insights gained into the molecular mechanisms of exosome-induced cell growth, angiogenesis, metastasis, immunosuppression, radiation resistance and chemotherapy resistance in the development and progression of NPC, as well as the clinical application of exosomes as diagnostic biomarkers and therapeutic agents. We also discuss the limitations and challenges in exosome application. We hope this review may provide some references for the use of exosomes in clinical intervention.
\end{abstract}

Key words: exosomes; NPC; angiogenesis; tumor metastasis

\section{Introduction}

NPC is a squamous cell carcinoma arising from the epithelial cells of the nasopharyngeal mucosa. Epstein-Barr virus (EBV) infection is one of the essential factors leading to NPC [1,2]. The geographic distribution of NPC is very skewed, mostly in southern China and southeast Asia, where NPC is still a major threat to people's life [3], despite the substantial improvements in large-scale screening and therapeutic strategies cut down the morbidity and mortality of the tumor [4]. NPC is not usually detected at the early stage and patients with advanced NPC are frequently accompanied by lymph node infiltration and distant metastasis. Some patients even develop resistance during radiotherapy or chemotherapy, which may lead to a very bad outcome $[5,6]$.

Exosomes are cell vesicles with a diameter of 40-100 nm, secreted by various types of cells outside the cell by fusing with the plasma membrane. The plasma membrane buds inward to form early endosomes, which then mature into late endosomes and multivesicular bodies (MVBs) with intraluminal vesicles. When late MVBs fuse with the plasma membrane, exosomes are released $[7,8]$. The content of exosomes consists of a variety of substances including lipids, nucleic acids and proteins specifically associated with the plasma membrane and cytoplasm. Exosomes involved in lipid metabolism have been found in plasma, urine, semen, saliva, etc. [9-11]. Exosomes can act as messengers to mediate cell communication and deliver the constituents to the recipient cells to perform crucial roles [12-14]. The uptake of exosomes is not random, but dependent upon the interaction between the protein on the surface of the exosomes and the recipient cells. Currently, exosomes are basically isolated by using ultra-high-speed centrifugation; other methods include size-based separation, polymer precipitation, immunoaffinity capture and the recent emergence of microphallus derivative technology [15]. Corresponding kits for exosome extraction have also been developed in recent years. Ultra-high-speed 
centrifugation technology has been known as the 'gold standard' for exosome isolation. The isolated exosomes are usually observed by transmission electron microscopy. Nano-tracking analysis is adopted to measure their size and western blotting is performed to trace the marker proteins such as CD9, CD63 or CD81 [16].

The poor prognosis of NPC is mainly attributed to insufficient consciousness of the prominent symptoms of the disease, the limited detections and therapeutic options [17]. In order to improve the prognosis of the patients, it is necessary to better understand the pathogenesis of NPC and to develop new therapeutic targets and effective strategies. Exosomes are of great significance in the occurrence and progression of NPC, which can be classified into EBV-related exosomes, exosomes derived from NPC cells or mesenchymal stem cells, and other types of exosomes [18]. The exosomal components enter the recipient cell through the membrane fusion with the target cell, and participate in many important physiological and pathological processes [19-23]. Originating from extracellular vesicles, exosomes have a unique source of cargo, capable of reproducing the molecular characteristics of parent cells in the nucleus [24]. There is growing evidence that different types of exosomes target their respective receptors to exert a variety of biological effects on NPC cell proliferation and function, resulting in promoting or suppressing the tumor growth [18,25-27]. In addition to serve as intercellular carriers for material transfer, exosomes can be utilized as biomarkers for disease diagnosis [28,29]. Exploring the role of exosomal molecules in the progression of NPC will facilitate to develop new biomarkers for the early diagnosis of NPC, as well as to provide novel treatment ideas to improve the therapeutic efficacy.

\section{Exosomes affect various processes of NPC}

Exosomes related to NPC have definite significance for the occurrence and progression of NPC. Exosomes carry cancer-aggravating or oppressing molecules that act on NPC or other stromal cells, which exert influence on different stages of NPC development and progression.

\section{NPC-related exosomes promote cell growth}

The onset of NPC is etiologically associated with EBV infection. The establishment of EBV latent infection in pre-invasive nasopharyngeal epithelium is considered to be an early stage of NPC pathogenesis. A mass of viral products are expressed during the latent phase of infection, including EB virus nuclear antigen (EBNA) 1, EB virus-encoded latent membrane proteins (LMP) 1 and 2, EB virus-encoded RNA (EBER), BamH I A rightward transcripts (BART), and some incubation period mRNA, with which EBV-related exosomes coexist $[30,31]$. LMP1 is predominantly produced in EBV infection, closely linked to the occurrence and growth of NPC. It has been shown to have multiple functions in vitro, covering promoting cell growth, protecting cells from apoptosis and enhancing cell motility [32]. LMP1 packaged by exosomes can activate normal fibroblasts and turn them into cancer-related fibroblasts through the key signaling pathway of nuclear factor (NF)-kB p65 [33]. Studies have revealed that LMP1 in NPC exosomes upregulates syndecan-2 (SDC2) and synaptotagmin-like-4 (SYTL4) via NF-kB signaling to stimulate the secretion of extracellular vesicles (EVs), promotes cell proliferation and tumor growth by activating ERK and AKT signal pathways and inducing vascular endothelial growth factor (VEGF) receptor expression [34,35]. BART1 miRNAs are thought to negatively regulate LMP1 expression, which may be in favor of NPC pathogenesis [32]. LMP2 can integrate into exosomes and then be released into the recipient cells [26]. In NPC patients, the expression of miR-24-3p, miR-891a, miR-106a-5p, miR-20a-5p and miR-1908 in sera and exosomes is significantly different from that in the healthy controls. These miRNAs have impacts on NPC cell proliferation and differentiation via downregulating MARK1 signal pathway [36]. Exosomes secreted by tumor cells and stromal cells are the key mediators of cell-to-cell communication in the tumor microenvironment, which facilitate tumor evolution and benefit other aspects of NPC aggravation [37].

\section{NPC-related exosomes mediate angiogenesis}

Angiogenesis is the process by which new blood vessels form. The formation of tumor blood vessels can enhance tumor growth, invasiveness and metastasis, leading to poor prognosis [38]. Tumor angiogenesis actually starts with tumor cells releasing molecules that send signals to promote blood vessel growth, causing local imbalances between factors that stimulate and inhibit angiogenesis [39]. The major contributors to tumor angiogenesis include the angiopoietin (Ang)/Tie-2(Tek) pathway, the VEGF family and its receptors. Mechanistically, angiogenesis is a critical event in NPC metastasis. Growing evidence suggests that exosomes derived from NPC can fine-tune endothelial cell characteristics to facilitate angiogenesis, especially under hypoxic conditions [40] (Figure 1). Quantitative proteomic analysis have demonstrated that the expression of angiogenic proteins, ICAM-1 and CD44v5, is up-regulated in the exosomes of NPC, while the expression of angiostatin TSP-1 is 
down-regulated [41]. As expected, these exosomes significantly contribute to the NPC angiogenesis. Some proteins for angiogenesis regulation are available in NPC tissues and exosomes. The glycolysis regulators of enzyme PFKFB3 and HS1-related protein X-1 (HAX1) can activate the ERK/AKT pathway and affect proliferation, migration, apoptosis and angiogenesis of the NPC cells [42,43]. Similarly, a large number of miRNAs from NPC-derived exosomes have been proven to participate in tumor angiogenesis. MiR-23a targets and inhibits testisspecific gene antigen (TSGA10, an anti-angiogenic factor), resulting in angiogenesis in NPC [44]. Highly expressed exosomal miR-17-5p encourages angiogenesis in NPC by targeting BAMB1 and regulating AKT/VEGF-A signaling [45]. Long noncoding RNA (lncRNA) CCAT2 from NPC-derived exosomes can promote angiogenesis [46]. EBV-related products in the exosomes of NPC can also affect tumor progression, such as the EBV-encoding RNAs (EBERs). For instance, EBERs regulate the expression of vascular cell adhesion molecule 1 (VCAM-1) through TLR3/RIG-I to induce angiogenesis [47]; EBV-miR-BART10-5p and miR-18a strongly promote angiogenesis and tumor growth of NPC by mediating the expression of VEGF and HIF1-a in a Spry3-dependent manner [48]. More important, the exosomal molecules not only have the ability to accelerate angiogenesis but also hold inhibitory functions. Juan $\mathrm{Lu}$, et al. have pointed out that overexpressed exosomal miR-9 inhibits angiogenesis and metastasis of NPC via targeting a pro-angiogenic protein of MDK and regulating PDK/AKT signal routing [49].

\section{NPC-related exosomes promote epithelial- mesenchymal transition (EMT) and metastasis}

NPC frequently metastasizes beyond the nasopharynx through additional blood vessels and lymph nodes, which is the most common reason for poor prognosis. EMT is a process by which the epithelial cells are transformed into quasi-mesenchymal cells. During this process, the interaction and interference between the cells and the extracellular matrix are reshaped with increased invasive and metastatic properties, resulting in separation of epithelial cells from each other and the basement membrane. Recent studies have revealed that EMT can enhance the mobility and invasiveness of the cell to gain greater metastatic potential, which prompts tumor metastasis and progression [50,51]. Matrix metalloproteinase 13 (MMP-13) in NPCrelated exosomes upregulates vimentin and reduces cadherin to facilitate tumor invasion, metastasis and angiogenesis; on the other hand, MMP13 expression is trans-activated by hypoxia-inducible factor a (HIF1a) [6,52]. Exosomes can mediate continuous interference between cancer cells and stromal cells and the effect becomes stronger under hypoxic conditions [53]. HIF1a is able to activate MMP13 and participate in cell metastasis itself, causing mutual change of EMT-related E/N cadherin expression [54]. The level of endogenous HIF1a can be enhanced by LMP1 while exosomal HIF1a is supportive for the pro-invasive potential of LMP1-positive exosomes associated with NPC [54,55]. The level of LMP1 is positively correlated with the expression of EMT markers and it functions to activate MMPs and miR-10b, inhibit miR-204, and consequently benefits the invasion and metastasis of NPC, whereas the invasion and metastasis process can be inhibited by miR-203. Moreover, LMP1 has been demonstrated to be able to stimulate the expression of miR-10b, which promotes cell migration and invasion via silencing HOXD10 and activating Twist [56]. Analogous to LMP1, LMP2 can also induce EMT-like changes in

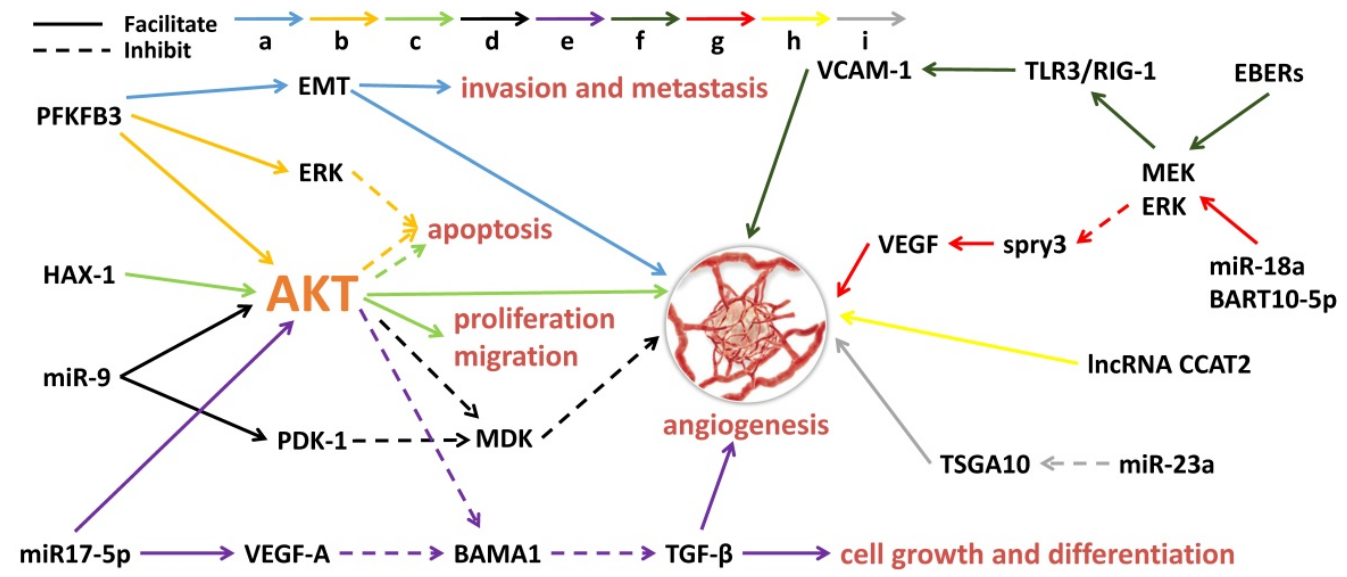

Figure 1. Exosomal molecules and their pathways involved in angiogenesis of NPC. 'a' to 'i' represents nine different molecular pathways. Multi-factors and pathways affect NPC angiogenesis, including some miRNAs, virus-related RNAs and proteins. The solid line indicates that the molecule can encourage the downstream signaling and the dotted line indicates inhibition. 
NPC cells [57]. The ERK1/2 pathway participates in raising the production of transcription factor Fra-1 and the level of MMP9; on the other hand, LMP2 and LMP1 phosphorylate 4EBP1 through the $\mathrm{PI} 3 \mathrm{~K} / \mathrm{Akt} / \mathrm{mTOR}$ signaling to elevate the expression of metastatic tumor antigen 1 (MTA1), thereby, expediting the invasiveness and metastatic ability of the NPC cells $[2,58,59]$. Fibroblast growth factor (FGF) 19 in the exosomes of NPC mesenchymal stem cells can encourage occurrence, proliferation and metastasis of the tumor and the mechanism involves activating the FGF19-FGFR4-dependent ERK signal cascade and regulating EMT to stimulate the growth of NPC [60]. PFKFB3 and HAX-1 can also strengthen the invasion and metastasis of NPC [42,43].

\section{NPC-related exosomes function in immune response}

The salient feature of NPC is the infiltration of a large number of non-malignant white blood cells in the primary tumor, mainly consisting of $\mathrm{T}$ lymphocytes and a small number of B cells, macrophages and dendritic cells, etc. However, this leukocyte infiltration has been found to disappear in the process of metastasis, replaced by rapidly and massively proliferated malignant cells with obvious anti-tumor immune feature [61]. Exosome-derived cytokines and substances, such as galectin-9 and CCL20, can induce local accumulation of regulatory $\mathrm{T}$ cells (Tregs) and promote NPC aggressiveness [62]. The immunomodulatory protein galactin-9 in EBV-infected NPC exosomes binds to ligand Tim-3, triggering apoptosis of mature CD4+ lymphocytes [63]. LMP1 can provoke galectin-9 expression, resulting in the release of exosomes containing LMP1 and galectin-9, and the recombinant of LMP1 with galectin-9 can induce strong inhibition on $\mathrm{T}$ cell proliferation in contrast to galectin 9 without LMP1 synergy [64]. Hypoxia is able to raise the level of miR-24-3p in NPC cells, sera and exosomes and enhance the inhibitory effects on $\mathrm{T}$ cell proliferation, Th1 and Th17 differentiation, as well as generate Tregs suppression via repressing FGF11 [65]. In addition, NPC exosomes have been observed to have the similar $\mathrm{T}$ cell inhibitory effect as the TW03 exosomes (EBV cell-negative or positive) do, by imposing on the pro-inflammatory cytokines [36]. IL-6 is a growth factor for a great deal of tumors. In NPC, exosomes have been demonstrated to enable to improve the production of IL-6 from macrophages to promote tumorigenesis [66]. As the core molecules that mediate immune suppression, Tregs can play tumor immune escape. It has been reported that exosomal chemokine CCL20 can recruit Tregs to tumor tissues, induce $\mathrm{T}$ cells to transform into inhibitory Tregs, and enhance Treg suppression [67]. In addition, gamma herpesvirus infection can change exosome protein contents in B cells [68]. Taking together, exosomal molecules can affect $\mathrm{T}$ cell activity, maintain continuous EBV infection and induce immunosuppression (Figure 2).

\section{NPC-related exosomes induce radiation resistance}

Radiotherapy is the major treatment for NPC, but radiation resistance seems inevitable in this process in some patients. Treatment failure of local radiotherapy accompanied with local recurrence or distant metastasis continues to be a severe challenge for NPC management $[69,70]$. Exosomes can arouse radioresistance by enhancing intercellular communication and cytotoxic damage in NPC cells [71]. Some molecules originated from NPC-related exosomes have been confirmed to be radiosensitive. It

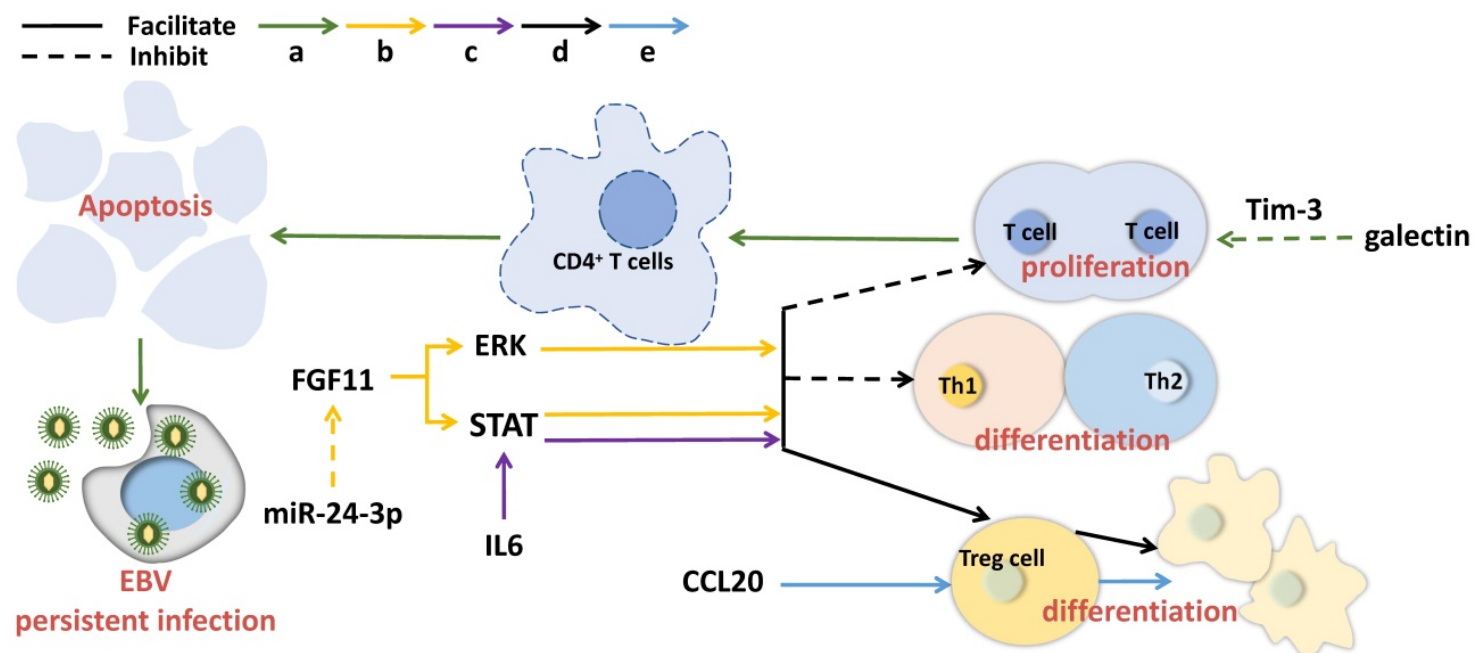

Figure 2. Exosomal molecules and their pathways involved in immunosuppression in NPC. Exosomal molecules can affect T cell activity, maintain continuous EBV infection and induce immunosuppression. The solid line indicates that the molecule can promote the downstream signaling and the dotted line represents inhibition. 
has been found that CircMYC, a newly discovered circular RNA (circRNA) in the circulating exosomes of NPC patients, is radioresistant and supportive for cell proliferation, and high level of circMYC is correlated with NPC recurrence [25]. Similarly, LMP1, as a vital cancer-promoting factor, exerts its carcinogenic effect by activating the P38 MARK signal of receptor cells to stimulate radiation resistance [72]. Studies have shown that different cell lines of NPC have different radiation sensitivity, of them, CNE2 being the strongest. Distinct from other cell lines, the secretory proteins expressed in CNE2 cells are mainly transported through non-classical pathways and exosomes [73]. In general, exosomes play a prominent role in transmitting radioresistance in NPC.

\section{NPC-related exosomes induce chemotherapy resistance}

In lung cancer exosomes, abnormal expression of some miRNAs and mRNAs regarding to cisplatin (DDP) sensitivity has been discovered in vitro, accompanied by decreased sensitivity of the tumor cells to cisplatin, and exosomes can transfer this DDP resistance to untreated cells to induce resistance [74]. NPC patients also develop resistance to drugs, where exosomes may be the transmitter. Doxorubicinresistant endothelial cells have been demonstrated to facilitate aggression, metastasis, EMT and chemoresistance in NPC via exosomes [75]. Overexpressed DDX53 has been detected in a variety of cancers and proven to be able to cause Taxol-resistance in cervix cancer cells in vitro through upregulating MDR1 [76]. Likewise, a latest study has reported that exosomes derived from Taxol-resistant NPC cells can transfer DDX53 resistance to the parental cells, while inhibiting the secretion of exosomes can block this process, thus revealing a new mechanism of drug resistance in NPC [77].

\section{NPC-related exosomes in clinical application}

Some exosomal molecules have multiple functions in the progression of NPC, such as LMP1 (Figure 3), and have been widely used, especially in clinical application for NPC diagnosis and treatment.

\section{Exosomes applied in NPC diagnosis}

Although the incidence of NPC has declined to a certain extent, its early detection is still a challenge due to its atypical symptoms and hidden location. Many NPC patients are already at an advanced stage at the time of diagnosis, worsening the outcome of the disease. Early diagnosis and interference are very important for NPC prognosis. Given that EBV is an influential issue for NPC, various EBV assays aiming at EBV-DNA and the relative antibody detection have been developed, but their sensitivity and specificity cannot satisfy the needs of the clinic [78,79]. Hence, exploring novel biomarkers and methods for early NPC diagnosis becomes exigent. As a non-invasive test, liquid biopsy, capable of detecting tumor cells, tumor-derived nucleic acids and exosomes in the circulating body fluid of patients, is believed to be more practical than the traditional tumor biopsy. Currently, the application of exosomes in cancer diagnosis and surveillance has aroused extensive attention $[80,81]$. The unique biogenesis of exosomes build up the ability to circulate freely in body fluids as various molecule carriers [82]. Many contemporary studies emphasize that biomolecules in NPC

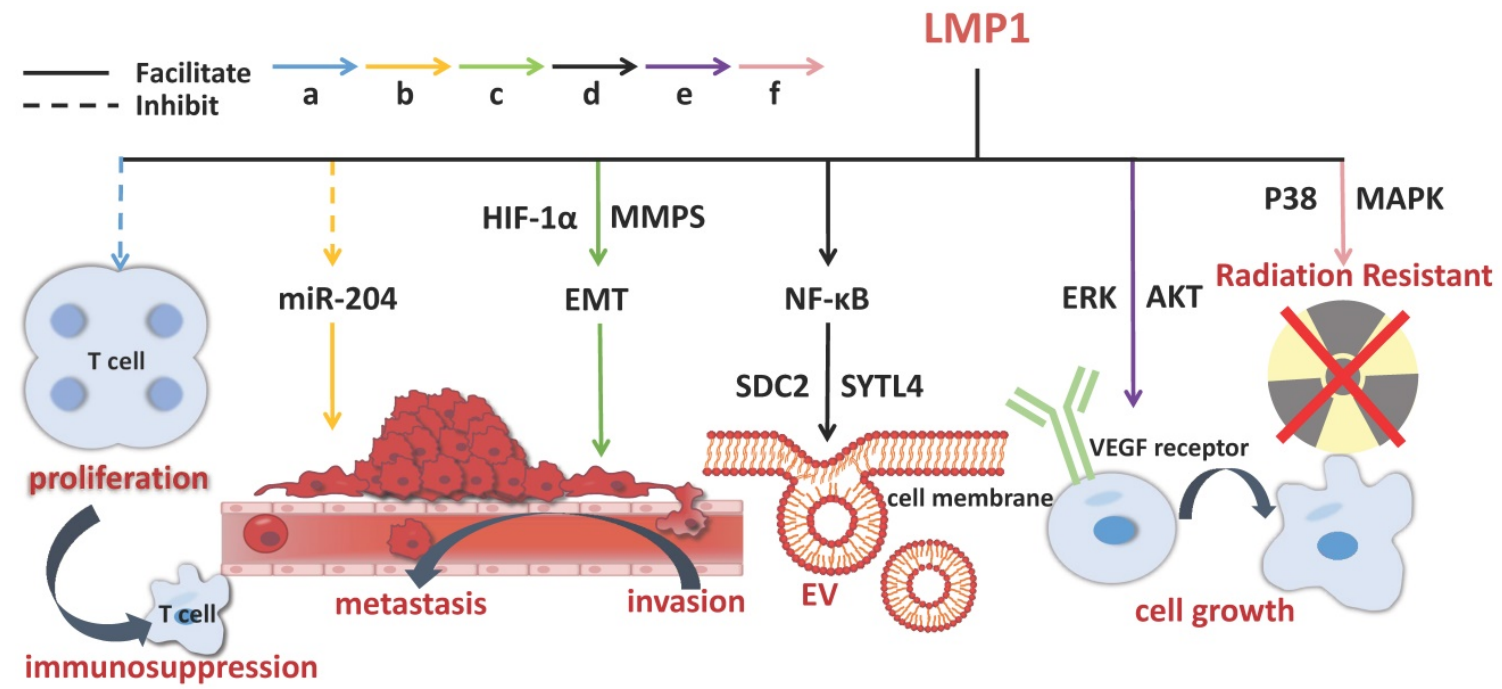

Figure 3. The molecular mechanisms of exosomal LMPI in NPC pathogenesis. LMPI promotes the progression of NPC through multiple channels, including stimulating EVs secretion, improving cell growth, prompting invasion, metastasis, and immunosuppression. The solid line indicates that the molecule can enhance the activity of its target and the dotted line indicates inhibition. 
exosomes can serve as novel diagnostic tumor biomarkers with the advanced technology platform focusing on nanoparticle identification. For example, Cytoflex, a new generation of flow cytometer equipped with $405 \mathrm{~nm}$ laser, has the ability to identify serum exosomes and microparticles [83,84]. NanoSight is an updated instrument, which can directly detect as small as $10 \mathrm{~nm}$ nanoparticles/exosomes and give a size-distribution graph [49]. EBV infection in NPC can induce differential expression of intracellular lncRNA in infected cells, exosomes and tumors, suggesting the potential clinical application of lncRNA as a biomarker [85]. Plasma and exosomal BART miRNAs produced by EBV-infected NPC cells have been identified as new indicators of NPC [86]. Exosomal circMYC has been proven to be correlative to radiation tolerance, and ROC analysis show that it has the potential to distinguish radiation tolerant NPC patients from the sensitive ones [25]. Exosomal molecules can be tested coupled with the existing EBV detection. Detection of cyclophilin A (CYPA) in sera and exosomes combined with EBV-VCA-IgA and LMP1 has been employed to diagnose NPC, where CYPA in exosomes exhibits a much higher level than that in whole sera [87]. In addition to be taken as biomarkers, exosomes have also been exploited to develop a type of exosomal nanovesicles in vivo as a contrast agent for $\mathrm{H}_{2} \mathrm{O}_{2}$-responsive catalytic photoacoustic imaging (PAI) in NPC, which achieves excellent lysosomal escape ability, strong stability and high sensitivity, better than the traditional non-biological materials for NPC detection [88]. Notably, the molecules on exosome surface such as CD9, CD63 and CD81 have been used to label the exosome to detect its secretion for tumor monitoring [89].

\section{Exosomes applied in NPC treatment}

Exosomes can provide protection for their contents against proteolytic digestion or drug action, which may be one of the causes for the failure of drug effect on NPC treatment, as exosomal cancerpromoting molecules move from the cancerous cells to the recipient cells. Increasingly, exosomes are being recognized as alternative therapeutics for NPC for their ability to induce potent cellular responses. For instance, targeting exosomal EBV-LMP1 transfer and miR-203 expression have positive significance for EBV-targeted therapy by aspirin in invasive NPC [90]. The commonly used targets for tumor control include PD-1/PD-L1, VEGF and EGFR. Substances used for immune escape therapy such as PD-1 and CTLA-4 can activate cytotoxic $\mathrm{T}$ cells but that action is frequently blocked, compromising the therapeutic effect [91].
Exosomes may offer solution to this issue because they have distinct advantages that uniquely position them as vehicles for the delivery of cancer drug when the molecules are included or linked to them with a ligand capable of accurately binding to their targets [92]. In NPC, a number of key regulatory molecules involved in the tumor pathological process have been identified and a few of them have been used for novel cancer immunotherapy in vitro or in vivo. A research team has worked to incorporate antagomiR targeting BART10-5p and miR-18a (which are intended to promote angiogenesis) into iRGD-tagged exosomes, resulting in preferentially suppressed angiogenesis and growth of NPC [48]. Delivering miR-34c (a tumor suppressor of NPC) to the cancerous cells through mesenchymal stem cell exosomes can impede $\beta$-chain protein and slow down the invasion, metastasis, proliferation and EMT of NPC cells, ultimately inhibiting tumor deterioration and radiation resistance [5].

\section{Limitations and challenges of exosomes in clinical applications}

Although researches on NPC exosomes have achieved certain accomplishments, there are also challenges and limitations in the application process.

The first lies in the means of detection. Despite the fact that tumor exosomes are attractive biomarkers for tumor diagnosis, their early detection for clinical use is still challenging. Some researchers have established a creative exosome detection method with high sensitivity and specificity, which refers to recombinase polymerase amplification triggered by adjacent ligation and transcription-mediated amplification (PAL-RPA-TMA) [93]; however, this approach requires innovative construction of DNA-labeled antibodies for specific detection targets (such as the proven biomarker LMP1). Based on hybrid chain reaction (HCR) and CRISPR-Cas12a double amplification technology, apta-HCR-CRISPR has been developed to directly examine extracellular vesicle proteins, which has attained high sensitivity [94]. An immunosorbent analysis adopting micro fluidic drop technology has been exploited to quantitatively identify exosomes [95]. Nonetheless, these detection methods are complicated, time consuming and costly, and more effective and feasible platform for exosome detection needs to be fulfilled.

The second involves the selective exosomal cargo loading mechanism driving biomolecules sorting into exosomes. Despite the entry of exosomes into recipient cells is non-specific, the exosome uptake mechanism depends on the recipient cells [96]. Studies have shown that seven miRNAs (let-7b-5p, miR-140-3p, miR-144-3p, miR-17-5p, miR-20a-5p, 
miR-20b-5p and miR-205-5p) are consistently upregulated in the plasma of patients with NPC; however, the plasma-derived exosomes yield the opposite outcome, indicating that miRNAs in plasma-derived exosomes have different expression mechanism from those in plasma [97,98]. The same phenomenon exists in the expression of viral miR-BART17 of EBV in NPC patients, where its concentration in plasma differs from that in plasma-derived exosomes [99]. It has been revealed that one of the key mechanisms for LMP1 to enter exosomes correlates to the C-terminal farnesylation of UCH-L1; inhibition of UCH-L1 deubiquitination activity exerts an anti-invasive effect on metastatic cancer cells [100,101]. CD63 has been found to be a specific protein on the surface of exosomes, which can bind to LMP1 and regulate LMP1 exosomal packaging $[102,103]$. Understanding the specific exosomal loading mechanism of how the NPC-related molecules selectively enter the recipient cells and how exosomes make cargo selection may benefit developing alternative policy to improve NPC diagnosis and treatment.

A study comparing the effect of conventional doxorubicin chemotherapy with the replacement therapy of Ag-TiO2-catalyzed reactive oxygen species generation in the treatment of 5-8F NPC cells has pointed out that beyond the classic chemotherapeutic agent, $\mathrm{Ag}-\mathrm{TiO} 2$ in the photo-catalytic process also exhibits cytostatic activity; tumor cell damage induced by cytostatic treatment enormously changes the number of released exosomes and leads to the predominance of tumor inhibitors in the exosomal miRNA profile [104]. Different treatment option has different impact on NPC progression and the involved mechanisms may be varied. The current strategies for NPC include radiotherapy, adjuvant chemotherapy, concurrent chemoradiotherapy and induction chemotherapy. Radiotherapy is classified into intensity-modulated radiation therapy (IMRT), intensity-modulated proton therapy (IMPT) and intensity-modulated carbon therapy (IMCT). The commonly used agents for chemotherapy are cisplatin and fluorouracil [3]. Probing the mechanisms of different therapeutic strategies and their effects on NPC-related exosomes will help break some new ground in dealing with NPC.

There are also limitations in exosome application. Due to the characteristics of stability, permeability, biocompatibility and extremely small size, exosomes can deliver drugs and nucleic acids as carriers $[105,106]$, however, most of them stay in the theoretical stage, far from practical applications [107]. The disadvantage of low drug loading rates and clinical production levels raise barriers for exosomes to target recipient cells with effectiveness and sufficiency. In addition, the non-tumor type specificity of exosomes in the body [108], and the protective effect of exosomes on their contents also restrain them to serve the clinic for precise tumor treatment.

\section{Conclusions}

With the improvement of diagnostic methods and treatment strategies, the incidence and prognosis of NPC have been improved, however, the mechanism of the tumor's development and progression is unclear, and problems emerge in practical treatment. The exosomes secreted from different types of NPC cells contain a variety of biomolecules, which participate in the circulation of body fluids, and affect the growth, angiogenesis, metastasis, immune response, radiation and chemotherapy sensitivity of NPC. As a promising non-invasive liquid biopsy biomarker, exosomes are highlighted in developing new diagnostic and treatment methods of NPC, but there are challenges in their clinical application. Conclusively, this article reviews the molecular mechanisms of NPC-related exosome-induced tumor progression in NPC and the potential clinical application of exosomes, which we hope may provide some references for the use of exosomes in clinical intervention.

\section{Abbreviations}

BART: BamHI A rightward transcripts; CCL20: C-C chemokine ligand 20; CD44v5: CD44 variant 5; DDP: cis-diamminedichloroplatinum(II); circRNA: circular RNA; CTLA-4: cytotoxic T lymphocyte associate protein-4; CYPA: cyclophilin A; EBER: Epstein-Barr virus-encoded RNA; EBNA: EpsteinBarr virus nuclear antigen; EBV: Epstein-Barr virus; EGFR: epidermal growth factor receptor; EMT: epithelial-mesenchymal transition; ERK: extracellular signal-regulated kinases; EV: extracellular vesicles; FGF: fibroblast growth factor; HAX-1: HS1-associated protein X-1; HCR: hybrid chain reaction; HIF1a: Hypoxia-inducible factor $a$; lncRNA: long non-coding RNA; ICAM-1: intercellular cell adhesion molecule-1; IMCT: intensity-modulated carbon therapy; IMPT: intensity-modulated proton therapy; IMRT: intensity-modulated radiation therapy; LMP: latent membrane proteins; MAPK: mitogen-activated protein kinase; MMP-13: matrix metalloproteinase 13; MTA1: metastatic tumor antigen 1; MVBs: multivesicular bodies; NPC: nasopharyngeal carcinoma; RIG-I: retinoic acid-inducible gene I; PAI: photoacoustic imaging; PD-1: programmed cell death protein 1; PD-L1: programmed cell death-ligand 1; PFKFB-3: enzymes 6-phosphofructo-2-kinase/ 
fructose-2,6-bisphosphatase-3; SDC2: syndecan-2; STAT: signal transducer and activator of transcription; SYTL: synaptophysin-like 4 ; TGF- $\beta$ : transforming growth factor- $\beta$; TLR3: toll-like receptor 3; Tim-3: T cell immunoglobulin and mucin domaincontaining protein 3; Tregs: regulatory $\mathrm{T}$ cells; TSGA10: testis-specific gene antigen 10; TSP-1: thrombospondin-1; UCH-L1: ubiquitin carboxylterminal hydrolase isozyme L1; VCAM-1: vascular cell adhesion molecule 1; VEGF: vascular endothelial growth factor.

\section{Competing Interests}

The authors have declared that no competing interest exists.

\section{References}

1. Tsao SW, Tsang CM, Lo KW. Epstein-Barr virus infection and nasopharyngeal carcinoma. Philosophical transactions of the Royal Society of London Series B, Biological sciences. 2017; 372: 20160270.

2. Raab-Traub N. Nasopharyngeal Carcinoma: An Evolving Role for the Epstein-Barr Virus. Current topics in microbiology and immunology. 2015; 390: 339-63.

3. Chen YP, Chan ATC, Le QT, Blanchard P, Sun Y, Ma J. Nasopharyngeal carcinoma. Lancet (London, England). 2019; 394: 64-80.

4. Tang LL, Chen WQ, Xue WQ, He YQ, Zheng RS, Zeng YX, et al. Global trends in incidence and mortality of nasopharyngeal carcinoma. Cancer letters. 2016; 374: 22-30

5. Wan FZ, Chen $\mathrm{KH}$, Sun $\mathrm{YC}$, Chen XC, Liang RB, Chen L, et al. Exosomes overexpressing miR-34c inhibit malignant behavior and reverse the radioresistance of nasopharyngeal carcinoma. Journal of translational medicine. 2020; 18: 12-30

6. Shan Y, You B, Shi S, Shi W, Zhang Z, Zhang Q, et al. Hypoxia-Induced Matrix Metalloproteinase-13 Expression in Exosomes from Nasopharyngeal Carcinoma Enhances Metastases. Cell death \& disease. 2018; 9: 382-94.

7. Xu R, Rai A, Chen M, Suwakulsiri W, Greening DW, Simpson RJ. Extracellular vesicles in cancer - implications for future improvements in cancer care. Nature reviews Clinical oncology. 2018; 15: 617-38.

8. Jeppesen DK, Fenix AM, Franklin JL, Higginbotham JN, Zhang Q, Zimmerman LJ, et al. Reassessment of Exosome Composition. Cell. 2019; 177: 428-45.e18.

9. Zhang J, Li S, Li L, Li M, Guo C, Yao J, et al. Exosome and exosomal microRNA: trafficking, sorting, and function. Genomics, proteomics \& bioinformatics. 2015; 13: 17-24.

10. Doyle LM, Wang MZ. Overview of Extracellular Vesicles, Their Origin, Composition, Purpose, and Methods for Exosome Isolation and Analysis. Cells. 2019; 8: 727-50.

11. Cheshmi B, Cheshomi H. Salivary exosomes: properties, medical applications, and isolation methods. Molecular biology reports. 2020; 47: 6295-307.

12. Wortzel I, Dror S, Kenific CM, Lyden D. Exosome-Mediated Metastasis: Communication from a Distance. Developmental cell. 2019; 49: 347-60.

13. Matarredona ER, Pastor AM. Extracellular Vesicle-Mediated Communication between the Glioblastoma and Its Microenvironment. Cells. 2019; 9: 96-108.

14. Whiteside TL. Exosome and mesenchymal stem cell cross-talk in the tumor microenvironment. Seminars in immunology. 2018; 35: 69-79.

15. Yang XX, Sun C, Wang L, Guo XL. New insight into isolation, identification techniques and medical applications of exosomes. Journal of controlled release official journal of the Controlled Release Society. 2019; 308: 119-29.

16. Théry C, Witwer KW, Aikawa E, Alcaraz MJ, Anderson JD, Andriantsitohaina R, et al. Minimal information for studies of extracellular vesicles 2018 (MISEV2018): a position statement of the International Society for Extracellular Vesicles and update of the MISEV2014 guidelines. Journal of extracellular vesicles. 2018; 7: 1535750

17. Lee HM, Okuda KS, González FE, Patel V. Current Perspectives on Nasopharyngeal Carcinoma. Advances in experimental medicine and biology. 2019; 1164: 11-34.

18. Zhou Y, Xia L, Lin J, Wang H, Oyang L, Tan S, et al. Exosomes in Nasopharyngeal Carcinoma. Journal of Cancer. 2018; 9: 767-77.

19. Wu Q, Zhou L, Lv D, Zhu X, Tang H. Exosome-mediated communication in the tumor microenvironment contributes to hepatocellular carcinoma development and progression. Journal of hematology \& oncology. 2019; 12: 53-64.

20. Koh E, Lee EJ, Nam GH, Hong Y, Cho E, Yang Y, et al. Exosome-SIRPa, a CD47 blockade increases cancer cell phagocytosis. Biomaterials. 2017; 121: $121-9$
21. Fu Y, Zhang L, Zhang F, Tang T, Zhou Q, Feng C, et al. Exosome-mediated miR-146a transfer suppresses type I interferon response and facilitates EV71 infection. PLoS pathogens. 2017; 13: e1006611.

22. Steinbichler TB, Dudás J, Riechelmann H, Skvortsova II. The role of exosomes in cancer metastasis. Seminars in cancer biology. 2017; 44: 170-81.

23. Syn N, Wang L, Sethi G, Thiery JP, Goh BC. Exosome-Mediated Metastasis: From Epithelial-Mesenchymal Transition to Escape from Immunosurveillance. Trends in pharmacological sciences. 2016; 37: 606-17.

24. Abels ER, Breakefield XO. Introduction to Extracellular Vesicles: Biogenesis, RNA Cargo Selection, Content, Release, and Uptake. Cellular and molecular neurobiology. 2016; 36: 301-12.

25. Luo Y, Ma J, Liu F, Guo J, Gui R. Diagnostic value of exosomal circMYC in radioresistant nasopharyngeal carcinoma. Head \& neck. 2020; 42: 3702-11.

26. Teow SY, Liew K, Khoo AS, Peh SC. Pathogenic Role of Exosomes in Epstein-Barr Virus (EBV)-Associated Cancers. International journal of biological sciences. 2017; 13: 1276-86.

27. Principe S, Hui AB, Bruce J, Sinha A, Liu FF, Kislinger T. Tumor-derived exosomes and microvesicles in head and neck cancer: implications for tumor biology and biomarker discovery. Proteomics. 2013; 13: 1608-23.

28. Inamdar S, Nitiyanandan R, Rege K. Emerging applications of exosomes in cancer therapeutics and diagnostics. Bioengineering \& translational medicine. 2017; 2: 70-80

29. Jin $\mathrm{H}, \mathrm{Wu} \mathrm{Y}$, Tan $\mathrm{X}$. The role of pancreatic cancer-derived exosomes in cancer progress and their potential application as biomarkers. Clinical \& translational oncology : official publication of the Federation of Spanish Oncology Societies and of the National Cancer Institute of Mexico. 2017; 19: 921-30.

30. Gallo A, Vella S, Miele M, Timoneri F, Di Bella M, Bosi S, et al. Global profiling of viral and cellular non-coding RNAs in Epstein-Barr virus-induced lymphoblastoid cell lines and released exosome cargos. Cancer letters. 2017; 388: 334-43.

31. Canitano A, Venturi G, Borghi M, Ammendolia MG, Fais S. Exosomes released in vitro from Epstein-Barr virus (EBV)-infected cells contain EBV-encoded latent phase mRNAs. Cancer letters. 2013; 337: 193-9.

32. Yoshizaki T, Kondo S, Wakisaka N, Murono S, Endo K, Sugimoto H, et al. Pathogenic role of Epstein-Barr virus latent membrane protein-1 in the development of nasopharyngeal carcinoma. Cancer letters. 2013; 337: 1-7.

33. Wu X, Zhou Z, Xu S, Liao C, Chen X, Li B, et al. Extracellular vesicle packaged LMP1-activated fibroblasts promote tumor progression via autophagy and stroma-tumor metabolism coupling. Cancer letters. 2020; 478: 93-106.

34. Meckes DG, Jr., Shair KH, Marquitz AR, Kung CP, Edwards RH, Raab-Traub $\mathrm{N}$. Human tumor virus utilizes exosomes for intercellular communication. Proceedings of the National Academy of Sciences of the United States of America. 2010; 107: 20370-5.

35. Liao C, Zhou Q, Zhang Z, Wu X, Zhou Z, Li B, et al. Epstein-Barr virus-encoded latent membrane protein 1 promotes extracellular vesicle secretion through syndecan- 2 and synaptotagmin-like- 4 in nasopharyngeal carcinoma cells. Cancer science. 2020; 111: 857-68.

36. Ye SB, Li ZL, Luo DH, Huang BJ, Chen YS, Zhang XS, et al. Tumor-derived exosomes promote tumor progression and T-cell dysfunction through the regulation of enriched exosomal microRNAs in human nasopharyngeal carcinoma. Oncotarget. 2014; 5: 5439-52.

37. Sun Z, Yang S, Zhou Q, Wang G, Song J, Li Z, et al. Emerging role of exosomederived long non-coding RNAs in tumor microenvironment. Molecular cancer. 2018; 17: 82-90

38. Ramjiawan RR, Griffioen AW, Duda DG. Anti-angiogenesis for cancer revisited: Is there a role for combinations with immunotherapy? Angiogenesis. 2017; 20: 185-204.

39. Ronca R, Benkheil M, Mitola S, Struyf S, Liekens S. Tumor angiogenesis revisited: Regulators and clinical implications. Medicinal research reviews. 2017; 37: 1231-74

40. Zhang L, Yu D. Exosomes in cancer development, metastasis, and immunity. Biochimica et biophysica acta Reviews on cancer. 2019: 1871: 455-68.

41. Chan YK, Zhang H, Liu P, Tsao SW, Lung ML, Mak NK, et al. Proteomic analysis of exosomes from nasopharyngeal carcinoma cell identifies intercellular transfer of angiogenic proteins. International journal of cancer. 2015; 137: 1830-41.

42. Gu M, Li L, Zhang Z, Chen J, Zhang W, Zhang J, et al. PFKFB3 promotes proliferation, migration and angiogenesis in nasopharyngeal carcinoma. ournal of Cancer. 2017: 8: 3887-96.

43. You B, Cao X, Shao X, Ni H, Shi S, Shan Y, et al. Clinical and biological significance of HAX-1 overexpression in nasopharyngeal carcinoma. Oncotarget. 2016; 7: 12505-24

44. Bao L, You B, Shi S, Shan Y, Zhang Q, Yue H, et al. Metastasis-associated miR-23a from nasopharyngeal carcinoma-derived exosomes mediates angiogenesis by repressing a novel target gene TSGA10. Oncogene. 2018; 37: 2873-89.

45. Duan B, Shi S, Yue H, You B, Shan Y, Zhu Z, et al. Exosomal miR-17-5p promotes angiogenesis in nasopharyngeal carcinoma via targeting BAMBI. Journal of Cancer. 2019; 10: 6681-92.

46. Zhou SK, Gao F, Zhong ZS, Yao H. [Long non-coding RNA colon cancer associated transcript-2 from nasopharyngeal carcinoma-derived exosomes promotes angiogenesis]. Zhonghua er bi yan hou tou jing wai ke za zhi = Chinese journal of otorhinolaryngology head and neck surgery. 2020; 55: 944-51. 
47. Cheng S, Li Z, He J, Fu S, Duan Y, Zhou Q, et al. Epstein-Barr virus noncoding RNAs from the extracellular vesicles of nasopharyngeal carcinoma (NPC) cells promote angiogenesis via TLR3/RIG-I-mediated VCAM-1 expression. Biochimica et biophysica acta Molecular basis of disease. 2019; 1865: 1201-13.

48. Wang J, Jiang Q, Faleti OD, Tsang CM, Zhao M, Wu G, et al. Exosomal Delivery of AntagomiRs Targeting Viral and Cellular MicroRNAs Synergistically Inhibits Cancer Angiogenesis. Molecular therapy Nucleic acids. 2020; 22: 153-65

49. Lu J, Liu QH, Wang F, Tan JJ, Deng YQ, Peng XH, et al. Exosomal miR-9 inhibits angiogenesis by targeting MDK and regulating PDK/AKT pathway in nasopharyngeal carcinoma. Journal of experimental \& clinical cancer research : CR. 2018; 37: 147-58.

50. Mittal V. Epithelial Mesenchymal Transition in Tumor Metastasis. Annual review of pathology. 2018; 13: 395-412.

51. Dongre A, Weinberg RA. New insights into the mechanisms of epithelial-mesenchymal transition and implications for cancer. Nature reviews Molecular cell biology. 2019; 20: 69-84.

52. You Y, Shan Y, Chen J, Yue H, You B, Shi S, et al. Matrix metalloproteinase 13-containing exosomes promote nasopharyngeal carcinoma metastasis. Cancer science. 2015; 106: 1669-77.

53. Meng W, Hao Y, He C, Li L, Zhu G. Exosome-orchestrated hypoxic tumor microenvironment. Molecular cancer. 2019; 18: 57-70.

54. Aga M, Bentz GL, Raffa S, Torrisi MR, Kondo S, Wakisaka N, et al. Exosomal HIF1a supports invasive potential of nasopharyngeal carcinoma-associated LMP1-positive exosomes. Oncogene. 2014; 33: 4613-22.

55. Huang SCM, Tsao SW, Tsang CM. Interplay of Viral Infection, Host Cell Factors and Tumor Microenvironment in the Pathogenesis of Nasopharyngeal Carcinoma. Cancers. 2018; 10: 106-23.

56. Li G, Wu Z, Peng Y, Liu X, Lu J, Wang L, et al. MicroRNA-10b induced by Epstein-Barr virus-encoded latent membrane protein-1 promotes the metastasis of human nasopharyngeal carcinoma cells. Cancer letters. 2010; 299: 29-36.

57. Kong QL, Hu LJ, Cao JY, Huang YJ, Xu LH, Liang Y, et al. Epstein-Barr virus-encoded LMP2A induces an epithelial-mesenchymal transition and increases the number of side population stem-like cancer cells in nasopharyngeal carcinoma. PLoS pathogens. 2010; 6: e1000940.

58. Lin Z, Wan X, Jiang R, Deng L, Gao Y, Tang J, et al. Epstein-Barr virus-encoded latent membrane protein $2 \mathrm{~A}$ promotes the epithelial-mesenchymal transition in nasopharyngeal carcinoma via metastatic tumor antigen 1 and mechanistic target of rapamycin signaling induction. Journal of virology. 2014; 88: 11872-85.

59. Lan YY, Hsiao JR, Chang KC, Chang JS, Chen CW, Lai HC, et al. Epstein-Barr virus latent membrane protein $2 \mathrm{~A}$ promotes invasion of nasopharyngeal carcinoma cells through ERK/Fra-1-mediated induction of matrix metalloproteinase 9. Journal of virology. 2012; 86: 6656-67.

60. Shi S, Zhang Q, Xia Y, You B, Shan Y, Bao L, et al. Mesenchymal stem cell-derived exosomes facilitate nasopharyngeal carcinoma progression. American journal of cancer research. 2016; 6: 459-72.

61. Kapetanakis NI, Baloche V, Busson P. Tumor exosomal microRNAs thwarting anti-tumor immune responses in nasopharyngeal carcinomas. Annals of translational medicine. 2017; 5: 164-7.

62. Gourzones C, Barjon C, Busson P. Host-tumor interactions in nasopharyngeal carcinomas. Seminars in cancer biology. 2012; 22: 127-36.

63. Klibi J, Niki T, Riedel A, Pioche-Durieu C, Souquere S, Rubinstein E, et al. Blood diffusion and Th1-suppressive effects of galectin-9-containing exosomes released by Epstein-Barr virus-infected nasopharyngeal carcinoma cells. Blood. 2009; 113: 1957-66

64. Keryer-Bibens $C$, Pioche-Durieu $C$, Villemant $C$, Souquère $S$, Nishi $N$, Hirashima $\mathrm{M}$, et al. Exosomes released by EBV-infected nasopharyngeal carcinoma cells convey the viral latent membrane protein 1 and the immunomodulatory protein galectin 9. BMC cancer. 2006; 6: 283-90.

65. Ye SB, Zhang $\mathrm{H}$, Cai TT, Liu YN, Ni JJ, He J, et al. Exosomal miR-24-3p impedes T-cell function by targeting FGF11 and serves as a potential prognostic biomarker for nasopharyngeal carcinoma. The Journal of pathology. 2016; 240: 329-40.

66. Wang X, Xiang Z, Tsao GS, Tu W. Exosomes derived from nasopharyngeal carcinoma cells induce IL-6 production from macrophages to promote tumorigenesis. Cellular \& molecular immunology. 2021; 8: 501-3.

67. Mrizak D, Martin N, Barjon C, Jimenez-Pailhes AS, Mustapha R, Niki T, et al. Effect of nasopharyngeal carcinoma-derived exosomes on human regulatory $\mathrm{T}$ cells. Journal of the National Cancer Institute. 2015; 107: 363-75.

68. Meckes DG, Jr, Gunawardena HP, Dekroon RM, Heaton PR, Edwards RH, Ozgur S, et al. Modulation of B-cell exosome proteins by gamma herpesvirus infection. Proceedings of the National Academy of Sciences of the United States of America. 2013; 110: E2925-33.

69. Zhan Y, Fan S. Multiple Mechanisms Involving in Radioresistance of Nasopharyngeal Carcinoma. Journal of Cancer. 2020; 11: 4193-204.

70. Lee AWM, Ng WT, Chan JYW, Corry J, Mäkitie A, Mendenhall WM, et al. Management of locally recurrent nasopharyngeal carcinoma. Cancer treatment reviews. 2019; 79: 101890

71. You B, Shan Y, Bao L, Chen J, Yang L, Zhang Q, et al. The biology and function of extracellular vesicles in nasopharyngeal carcinoma (Review). International journal of oncology. 2018; 52: 38-46.
72. Zhang Z, Yu X, Zhou Z, Li B, Peng J, Wu X, et al. LMP1-positive extracellular vesicles promote radioresistance in nasopharyngeal carcinoma cells through P38 MAPK signaling. Cancer medicine. 2019; 8: 6082-94.

73. Chen ZT, Li L, Guo Y, Qu S, Zhao W, Chen H, et al. Analysis of the differential secretome of nasopharyngeal carcinoma cell lines CNE-2R and CNE-2. Oncology reports. 2015; 34: 2477-88.

74. Xiao X, Yu S, Li S, Wu J, Ma R, Cao H, et al. Exosomes: decreased sensitivity of lung cancer A549 cells to cisplatin. PloS one. 2014; 9: e89534.

75. Huang L, Hu C, Chao H, Zhang Y, Li Y, Hou J, et al. Drug-resistant endothelial cells facilitate progression, EMT and chemoresistance in nasopharyngeal carcinoma via exosomes. Cellular signalling. 2019; 63: 109385.

76. Park SY, Kim WJ, Byun JH, Lee JJ, Jeoung D, Park ST, et al. Role of DDX53 in taxol-resistance of cervix cancer cells in vitro. Biochemical and biophysical research communications. 2018; 506: 641-7.

77. Yuan F, Zhou ZF. Exosomes derived from Taxol-resistant nasopharyngeal carcinoma (NPC) cells transferred DDX53 to NPC cells and promoted cancer resistance to Taxol. European review for medical and pharmacological sciences. 2021; 25: 127-38.

78. AbuSalah MAH, Gan SH, Al-Hatamleh MAI, Irekeola AA, Shueb RH, Yean Yean C. Recent Advances in Diagnostic Approaches for Epstein-Barr Virus. Pathogens (Basel, Switzerland). 2020; 9: 226-42.

79. Tan R, Phua SKA, Soong YL, Oon LLE, Chan KS, Lucky SS, et al. Clinical utility of Epstein-Barr virus DNA and other liquid biopsy markers in nasopharyngeal carcinoma. Cancer communications (London, England). 2020; 40: 564-85.

80. Jalalian SH, Ramezani M, Jalalian SA, Abnous K, Taghdisi SM. Exosomes, new biomarkers in early cancer detection. Analytical biochemistry. 2019; 571: 1-13.

81. Vaidyanathan R, Soon RH, Zhang P, Jiang K, Lim CT. Cancer diagnosis: from tumor to liquid biopsy and beyond. Lab on a chip. 2018; 19: 11-34.

82. Whiteside TL. The potential of tumor-derived exosomes for noninvasive cancer monitoring: an update. Expert review of molecular diagnostics. 2018; 18: $1029-40$

83. Lucchetti D, Battaglia A, Ricciardi-Tenore C, Colella F, Perelli L, De Maria R, et al. Measuring Extracellular Vesicles by Conventional Flow Cytometry: Dream or Reality? International journal of molecular sciences. 2020; 21: 6257-71.

84. Ender F, Zamzow P, Bubnoff NV, Gieseler F. Detection and Quantification of Extracellular Vesicles via FACS: Membrane Labeling Matters! International journal of molecular sciences. 2019; 21: 291-303.

85. Zhang J, Li X, Hu J, Cao P, Yan Q, Zhang S, et al. Long noncoding RNAs involvement in Epstein-Barr virus infection and tumorigenesis. Virology journal. 2020; 17: 51-8.

86. Gourzones C, Gelin A, Bombik I, Klibi J, Vérillaud B, Guigay J, et al. Extracellular release and blood diffusion of BART viral micro-RNAs produced by EBV-infected nasopharyngeal carcinoma cells. Virology journal. 2010; 7: 271-82.

87. Liu L, Zuo L, Yang J, Xin S, Zhang J, Zhou J, et al. Exosomal cyclophilin A as a novel noninvasive biomarker for Epstein-Barr virus associated nasopharyngeal carcinoma. Cancer medicine. 2019; 8: 3142-51.

88. Ding H, Cai Y, Gao L, Liang M, Miao B, Wu H, et al. Exosome-like Nanozyme Vesicles for $\mathrm{H}(2) \mathrm{O}(2)$-Responsive Catalytic Photoacoustic Imaging of Xenograft Nasopharyngeal Carcinoma. Nano letters. 2019; 19: 203-9.

89. Pietrowska M, Funk S, Gawin M, Marczak $€$, Abramowicz A, Widłak P, et al. Isolation of Exosomes for the Purpose of Protein Cargo Analysis with the Use of Mass Spectrometry. Methods in molecular biology (Clifton, NJ). 2017; 1654: 291-307.

90. Zuo L, Xie Y, Tang J, Xin S, Liu L, Zhang S, et al. Targeting Exosomal EBV-LMP1 Transfer and miR-203 Expression via the NF-KB Pathway: The Therapeutic Role of Aspirin in NPC. Molecular therapy Nucleic acids. 2019; 17: $175-84$

91. Wei SC, Duffy CR, Allison JP. Fundamental Mechanisms of Immune Checkpoint Blockade Therapy. Cancer discovery. 2018; 8: 1069-86.

92. Hofmann L, Ludwig S, Vahl JM, Brunner C, Hoffmann TK, Theodoraki MN. The Emerging Role of Exosomes in Diagnosis, Prognosis, and Therapy in Head and Neck Cancer. International journal of molecular sciences. 2020; 21: 4072-93.

93. Liu W, Li J, Wu Y, Xing S, Lai Y, Zhang G. Target-induced proximity ligation triggers recombinase polymerase amplification and transcription-mediated amplification to detect tumor-derived exosomes in nasopharyngeal carcinoma with high sensitivity. Biosensors \& bioelectronics. 2018; 102: 204-10.

94. Xing S, Lu Z, Huang Q, Li H, Wang Y, Lai Y, et al. An ultrasensitive hybridization chain reaction-amplified CRISPR-Cas12a aptasensor for extracellular vesicle surface protein quantification. Theranostics. 2020; 10: $10262-73$

95. Liu C, Xu X, Li B, Situ B, Pan W, Hu Y, et al. Single-Exosome-Counting Immunoassays for Cancer Diagnostics. Nano letters. 2018; 18: 4226-32.

96. Horibe S, Tanahashi T, Kawauchi S, Murakami Y, Rikitake Y. Mechanism of recipient cell-dependent differences in exosome uptake. BMC cancer. 2018; 18: $47-55$

97. Zou X, Zhu D, Zhang H, Zhang S, Zhou X, He X, et al. MicroRNA expression profiling analysis in serum for nasopharyngeal carcinoma diagnosis. Gene. 2020; 727: 144243 .

98. Zhang H, Zou X, Wu L, Zhang S, Wang T, Liu P, et al. Identification of a 7-microRNA signature in plasma as promising biomarker for nasopharyngeal carcinoma detection. Cancer medicine. 2020; 9: 1230-41. 
99. Gourzones C, Ferrand FR, Amiel C, Vérillaud B, Barat A, Guérin M, et al. Consistent high concentration of the viral microRNA BART17 in plasma samples from nasopharyngeal carcinoma patients--evidence of non-exosomal transport. Virology journal. 2013; 10: 119-30.

100. Kobayashi E, Hwang D, Bheda-Malge A, Whitehurst CB, Kabanov AV, Kondo $\mathrm{S}$, et al. Inhibition of UCH-L1 Deubiquitinating Activity with Two Forms of LDN-57444 Has Anti-Invasive Effects in Metastatic Carcinoma Cells. International journal of molecular sciences. 2019; 20: 3733-50.

101. Kobayashi E, Aga M, Kondo S, Whitehurst C, Yoshizaki T, Pagano JS, et al. C-Terminal Farnesylation of UCH-L1 Plays a Role in Transport of Epstein-Barr Virus Primary Oncoprotein LMP1 to Exosomes. mSphere. 2018; 3: e00030-18.

102. Nkosi D, Howell LA, Cheerathodi MR, Hurwitz SN, Tremblay DC, Liu X, et al. Transmembrane Domains Mediate Intra- and Extracellular Trafficking of Epstein-Barr Virus Latent Membrane Protein 1. Journal of virology. 2018; 92: 00280-18.

103. Hurwitz SN, Nkosi D, Conlon MM, York SB, Liu X, Tremblay DC, et al. CD63 Regulates Epstein-Barr Virus LMP1 Exosomal Packaging, Enhancement of Vesicle Production, and Noncanonical NF-kB Signaling. Journal of virology. 2017; 91: e02251-16.

104. Harmati M, Tarnai Z, Decsi G, Kormondi S, Szegletes Z, Janovak L, et al. Stressors alter intercellular communication and exosome profile of nasopharyngeal carcinoma cells. Journal of oral pathology \& medicine : official publication of the International Association of Oral Pathologists and the American Academy of Oral Pathology. 2017; 46: 259-66.

105. Arrighetti N, Corbo C, Evangelopoulos M, Pastò A, Zuco V, Tasciotti E. Exosome-like Nanovectors for Drug Delivery in Cancer. Current medicinal chemistry. 2019; 26: 6132-48.

106. Pullan JE, Confeld MI, Osborn JK, Kim J, Sarkar K, Mallik S. Exosomes as Drug Carriers for Cancer Therapy. Molecular pharmaceutics. 2019; 16: 1789-98.

107. Tang Z, Li D, Hou S, Zhu X. The cancer exosomes: Clinical implications, applications and challenges. International journal of cancer. 2020; 146: 2946-59.

108. Ko YH. EBV and human cancer. Experimental \& molecular medicine. 2015; 47: e130. 